\title{
AN UPGRADED ATLAS CENTRAL TRIGGER FOR 2014 LUMINOSITIES
}

\section{Overview}

Since march 2012 the LHC is running at a center of mass energy of $8 \mathrm{TeV}$, has reached instantaneous luminosities of $6.7 \times 10^{33} \mathrm{~cm}^{-2} \mathrm{~s}^{-1}$ and produced events with up to 40 interactions per collision. These conditions place stringent operational and physical requirements on the trigger system.

In 2014, the LHC will run at $14 \mathrm{TeV}$ and the luminosity will exceed $10^{34} \mathrm{~cm}^{-2} \mathrm{~s}^{-1}$. With higher luminosities, the required number and complexity of Level-1 triggers will increase in order to satisfy the physics goals of ATLAS while keeping the total Level-1 rates below $100 \mathrm{kHz}$.

\section{The ATLAS Trigger System}

The trigger system of the ATLAS detector is structured in a 3-level architecture in order to subsequently reduce the event rate from the $\sim 1$ $\mathrm{GHz}$ interaction rate to a few $100 \mathrm{~Hz}$ recorded on tape. The first level operates hardware based and reduces the rate to $75 \mathrm{kHz}$ maximum with a latency of $2.5 \mu \mathrm{s}$. Its core unit is the Central Trigger Processor (CTP). Adjacently, the HighLevel Trigger (HLT) software refines the trigger decision, using higher granularity read-out information.

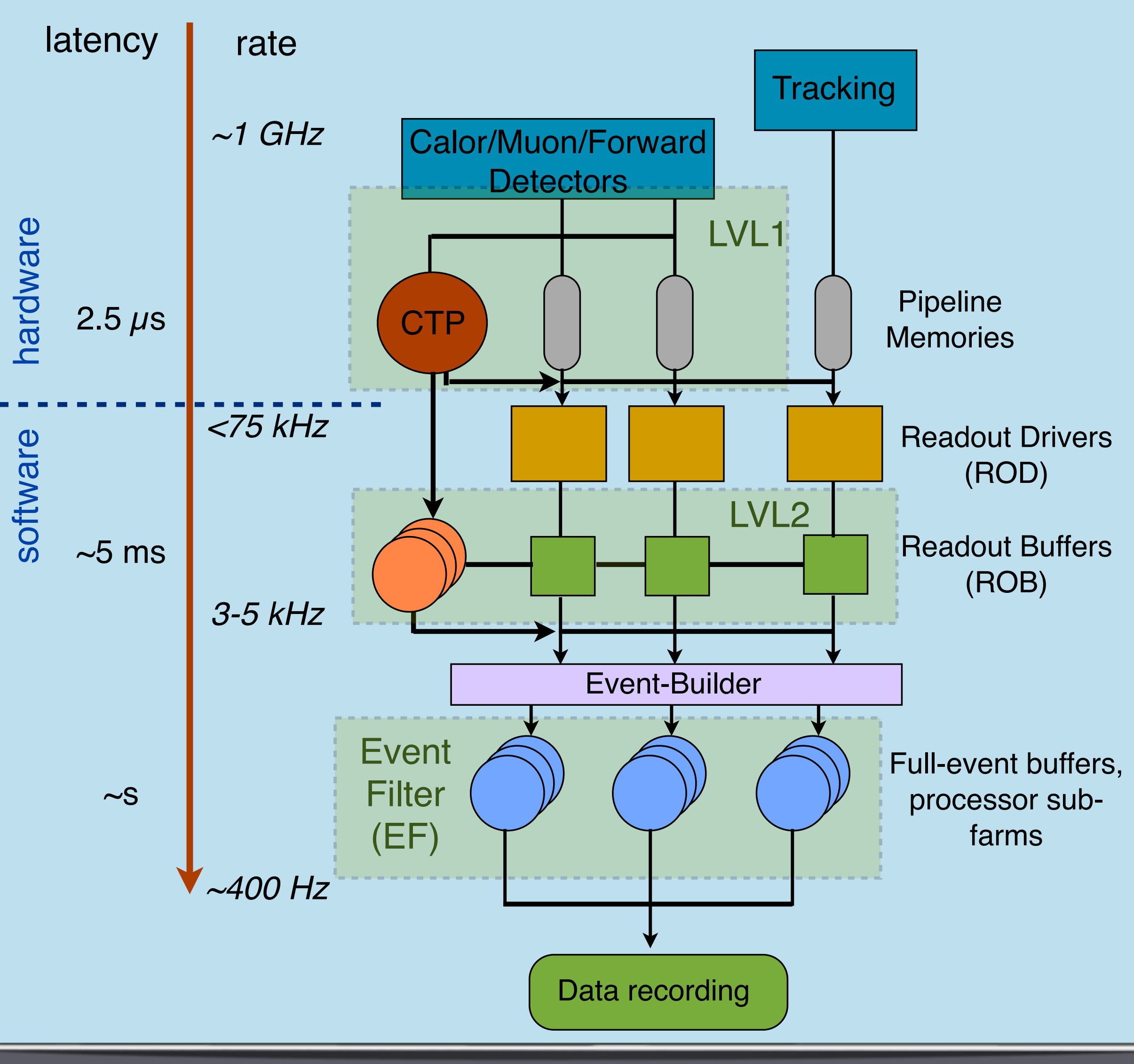

The Central Trigger Processor

is implemented in custom-built electronics. There are 3 CTPIN boards, each of which accepts $4 \times 31$ inputs that are synchronised, aligned and monitored with scalers. 160 input signals are selected by a switch matrix and transmitted via the Pattern In Time (PIT) bus to the CTPCORE board, which forms the actual level- 1 accept (L1A), and to the CTPMON board for per-bunch monitoring. Four CTPOUT modules fan out the L1A and timing information to the Trigger, Timing and Control (TTC) partitions of the subdetectors. The CTPMI module, the interface to the LHC, is responsible for the distribution of timing signals. The sub-detectors can send calibration requests to the CTPCAL module.

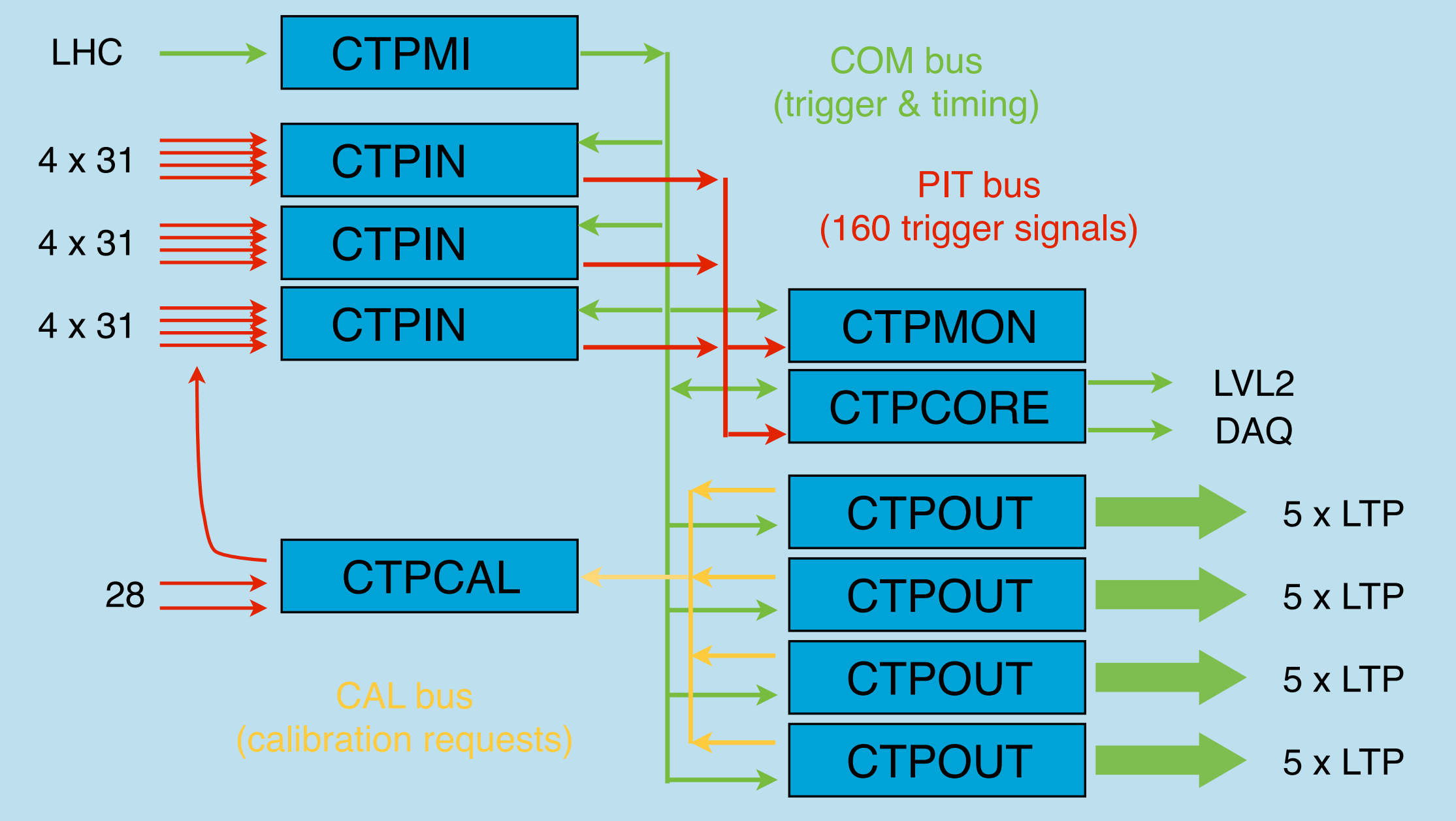

HC Machine.

References

L. Evans and P. Bryant (editors). JINST 3 (2008) S0800 The ATLAS Experiment at the CERN Large Hadron Collider. The ATLAS Collaboration. JINST 3 (2008) S08003 S Ask et al 2008 JINST 3 P08002

SLAS high-level trigger, data-acquisition and controls: Technical Deign Report, Jenni, P. et al 2003 ATLAS Technical Design Report

\section{Status Quo}

The CTP provides a variety of functionalities:

synchronisation/distribution of LHC timing signals (40 MHz clock (BC), Orbit )

combining information from calorimeter

muon trigger processors, forward detectors

formation of 256 trigger menu items

(flexible logical combination)

generation of level-1 trigger decision (L1A)

bunch group masking ( 8 bunch groups)

prescaling of trigger items

2 types of deadtime: simple and complex busy handling

summary information to level 2

readout data to $D A Q$

monitoring of trigger rates, deadtime, busy information

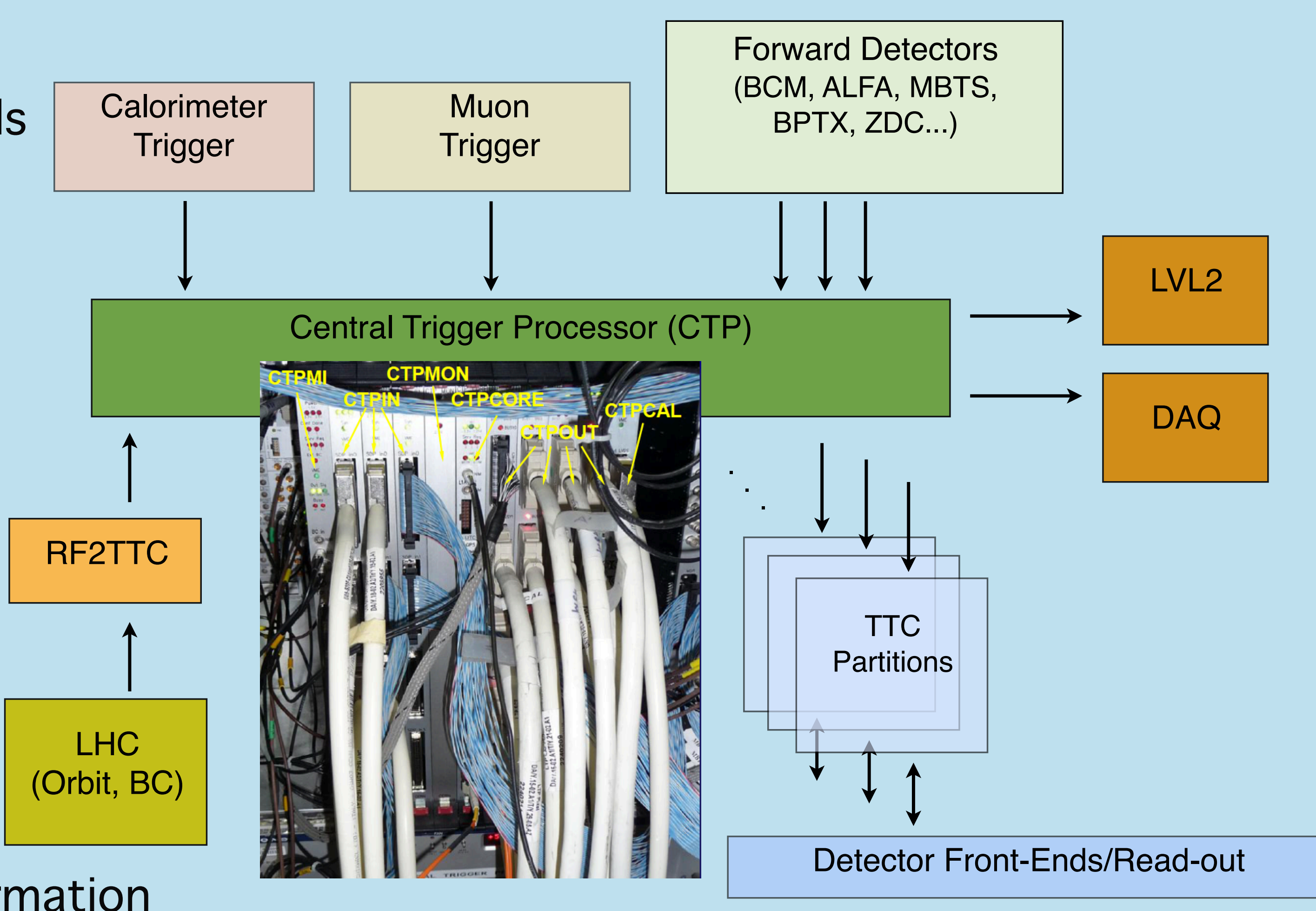

Run 200498 : CTPIN Counter Rates, $\mathrm{Hz}$

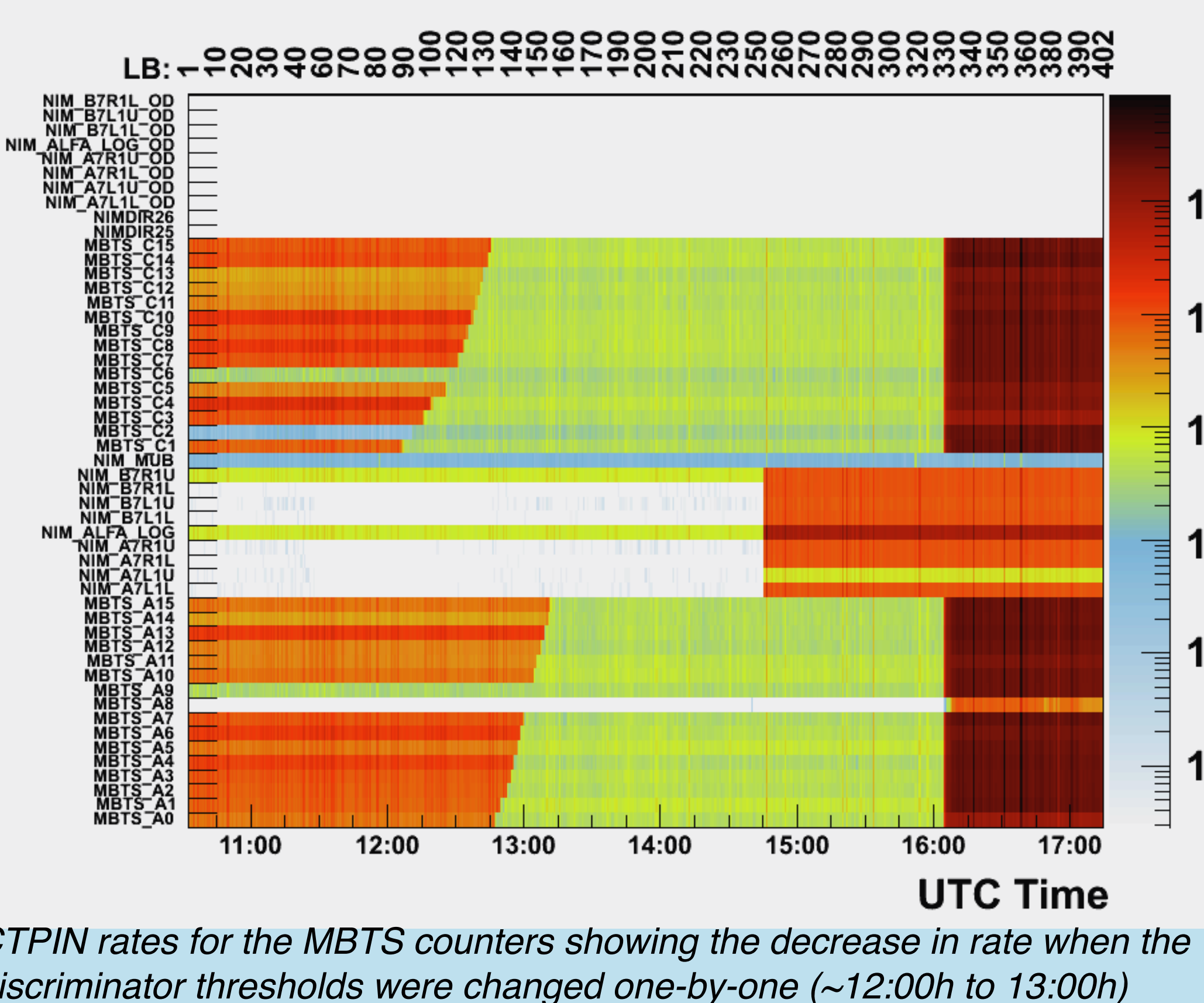

The CTP operation during the first years of data taking has been very smooth: The availability of the central trigger system was close to $100 \%$. Nevertheless, there are improvements ongoing constantly in order to get a more detailed picture of the system during runtime and to detect and identify problems as fast as possible and prevent them in future. Some examples of recently implemented additional features are

LHC-turn counter to detect missed orbit signal

monitoring of irregular orbit lengths

cross checks of bunch crossing IDs

simplifications in configuration (e.g. dead time settings)

The CTP group has to interact closely with other systems and consider their requests. One example are the hardware settings of the Minimum Bias Trigger Scintillators (MBTS), which had to be re-tuned at the beginning of the year for a special run, to compensate for aging effects of the scintillators. The discriminator thresholds of the photo-multiplier read-out were adjusted to reduce the background rate to tolerable level.

\section{Upgrade Plans for 2014}

Some of the CTP features are operated at the limits of their capacity. The primary motivation for the Phase- 0 upgrade during the shutdown of $2013 / 14$ is the removal of these resource limitations. The currently planned steps are

double trigger inputs on PIT bus double trigger items

12 optical inputs

improved bunch group masking

improved trigger item monitoring

4 partitions:

1 primary for L1A

3 secondary for commissioning

While the electrical PIT bus will be kept but used with double data rate, the other upgrade plans require the redesign of the CTPCORE module, the COM backplane and the CTPOUT modules. The CTPCORE++ module

\begin{tabular}{|c|c|c|c|}
\hline from a recent trigger menu & Used & Available & Upgrade \\
\hline CTPIN input Cables (partially used) & 9 & 12 & \\
\hline CTPIN input Signals & 212 & 372 & \\
\hline CTPIN integrating monitoring counters & 138 & 928 & 1856 \\
\hline CTP PIT bus lines & 160 & 160 & 320 \\
\hline CTPCORE trigger items & 241 & 256 & 512 \\
\hline CTPCORE bunch groups & 8 & 8 & 16 \\
\hline CTPCORE max. number of AND terms & 6 & 256 & 512 \\
\hline CTPCORE max. number of bits in OR terms & 6 & 12 & 15 \\
\hline CTPCORE per-bunch trigger item counters & 12 & 12 & 256 \\
\hline CTPMON per-bunch monitoring counters & 88 & 160 & \\
\hline CTPOUT output cables to TTC partitions & 20 & 20 & 25 \\
\hline
\end{tabular}
or upgraded sub-systems. It will be capable of the formation of 512 trigger items and will have 256 per-bunch counters for the trigger item monitoring available. The number of bunch groups will be doubled to 16 and the masking will be applied after the item formation instead of being part of the item.

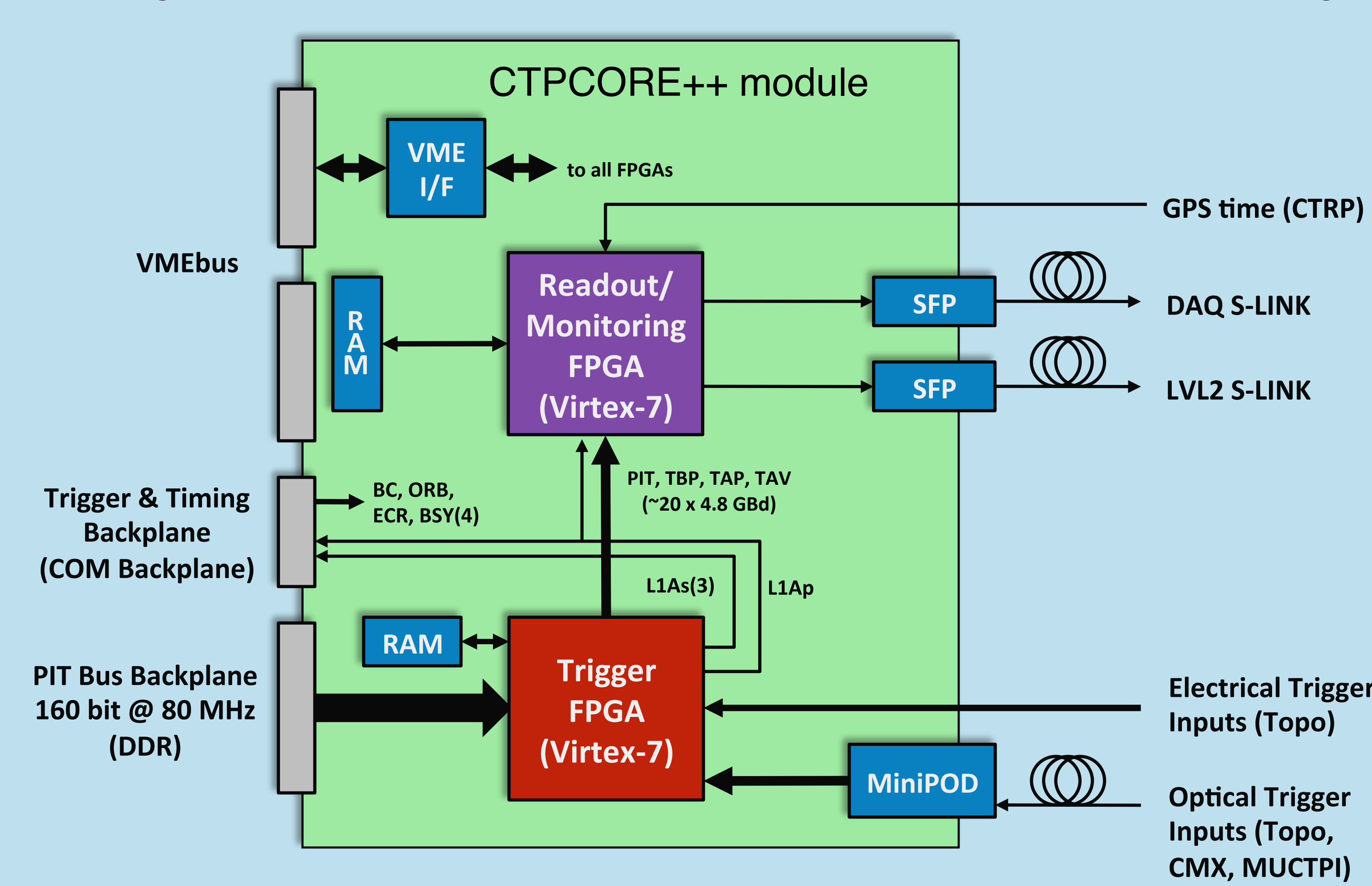

include the partitioning of the L1A generation, where all partitions share a common trigger menu and timing. There will be 4 partitions: One primary - or "physics" - partition, which is the only one providing information for LVL2 and DAQ read-out, and 3 secondary partitions that have their own selec tion of trigger items and deadtime handling and will be used for detector commissioning, calibration, etc. The existing COM backplane allows for the implementation of two partitions and is hence foreseen to be replaced to accommodate four. It could then allow also for one more CTPOUT module which would provide the possibility to increase the number of TTC partitions. The CTPOUT modules themselves will have to be adapted to these changes and the new CTPOUT++ modules in addition can be used for a per-bunch busy monitoring.

The aim is to install the upgraded CTP in time to be ready for detector commissioning in Q1'2014. 